\title{
Is There a Role for Intraoperative Radiation Therapy in Patients with Resected Pancreatic Adenocarcinoma?
}

\author{
Jeffrey J. Meyer, MD and Christopher H. Crane, MD \\ Department of Radiation Oncology, Unit 97, U.T. M.D. Anderson Cancer Center, Houston, TX
}

The value of adjuvant radiotherapy is well established in many tumor sites and is commonly used when pathologic features of a patient's primary tumor place them at sufficiently high risk for local-regional recurrence to warrant additional intervention. The rationale for the use of adjuvant radiotherapy in such situations is the traditional shared goal of the surgical and radiation oncologist: to optimize local-regional control. Attaining local-regional control prevents the morbidity of a local recurrence, and, in some situations, improves overall survival, perhaps best exemplified in the case of postmastectomy radiotherapy for women with high-risk breast cancer. ${ }^{1}$ For pancreatic cancer management, adjuvant radiation has been delivered through fractionated external beam radiation treatments (EBRT) and/or single-fraction high-dose intraoperative radiation therapy (IORT). IORT has the benefit of directly moving critical normal tissues of the upper abdomen away from the radiation beam. ${ }^{2}$

Prevention of local recurrence leading to an improvement in survival may result through a number of mechanisms. The most straightforward of these is the underappreciated fact that local recurrence, particularly at certain sites such as the head and neck, can itself lead to disease-specific mortality. Also, radiation can sterilize remaining cells able to proliferate at the local site and then metastasize, causing eventual death; this may be all the more important in light of evidence suggesting that cells can be modulated by the postoperative microenvironment to yield more a more aggressive phenotype, as has recently been suggested in breast cancer. ${ }^{3-5}$ Another, highly intriguing, possible effect is for irradiation of local tumor

(C) Society of Surgical Oncology 2009

Published Online: 13 June 2009

C. H. Crane, MD

e-mail: ccrane@mdanderson.org cells to induce abscopal, immune-related killing effects on distant sites of micrometastases. ${ }^{5,6}$

Attaining local-regional control is all the more paramount when patterns of cancer failure are predominantly local-regional and/or reasonably effective chemotherapy is available to treat systemic micrometastatic disease but not sterilize local-regional disease. Therein lies the controversy over adjuvant radiotherapy (be it EBRT or IORT) for resectable pancreatic adenocarcinoma. Although clinical and autopsy series have revealed local-regional (tumor bed and regional lymph nodes) failure to be a common event (50\% or greater) following pancreaticoduodenectomy, it is much less common for these failures to be in isolation with respect to distant disease and also for the local-regional failure itself to be the clear proximate cause of death. ${ }^{7-9}$

Acknowledging the high rates of distant failure (liver, peritoneal cavity), the value of primary radical surgery in patients with resectable disease may itself be questioned. ${ }^{10}$ Two randomized trials provide evidence that surgery does improve long-term survival rates compared with bypass intervention or primary chemotherapy and concurrent radiation. ${ }^{11,12}$ However, true long-term disease-free survivorship (apparent cure) is uncommon with surgery alone, and the need for effective systemic therapies is obvious. The recently reported CONKO-001 trial showed that gemcitabine following surgery delays disease failure and also likely provides higher long-term survival rates (estimated 5-year disease-free survival of $16 \%$ vs $6.5 \%$ with surgery alone). ${ }^{13}$ Whether additional locally directed therapies beyond surgery add meaningful benefit -in the context of contemporary systemic therapy optionsremains an open question. Although the role of adjuvant chemoradiotherapy was established in an early Gastrointestinal Tumor Study Group trial and through retrospective institutional reports, ${ }^{14,15}$ the actual value of the radiation component has been called into question, most recently by the controversial ESPAC results. ${ }^{16}$ 
What are the data regarding the benefits and toxicities of $\mathrm{IORT} \pm \mathrm{EBRT}$ for resectable pancreatic cancer? In this issue of the Annals of Surgical Oncology, investigators from the Thomas Jefferson University departments of Radiation Oncology and Surgery report their experience with 83 patients with resected pancreatic cancer treated between 1995 and 2005. Of these, 37 patients were treated with IORT; about $75 \%$ of this group also received EBRT, and $84 \%$ received adjuvant chemotherapy. In the group treated with resection but without IORT, about two-thirds underwent adjuvant EBRT and chemotherapy. IORT did not significantly increase perioperative complication rates. As patients were not randomized to the use of IORT, the authors used a propensity score adjustment in their analysis of disease control to account for differing prognostic factors between the two groups. Although IORT was associated with an odds ratio of 0.41 for local-regional recurrence, this reduction did not reach statistical significance $(P=.23)$. The confidence intervals for this ratio were quite large (0.10-10.30), reflecting patient numbers. IORT was not associated with improved survival.

Institutional reports and a randomized trial have shown surgery followed by IORT to yield lower local-regional recurrence rates compared with surgery alone, although a positive impact on survival has not been readily demonstrated. ${ }^{17-19}$ As the authors appropriately address, improvements in systemic therapy are generally necessary before prevention of local recurrence translates into a survival benefit. $^{20}$ As long as the best systemic therapy options provide only modest control of distant disease, local tumor control improvements will not translate into a survival benefit. Finally, the emergence of novel chemotherapeutic or targeted agents that impact distant metastases may also eventually impact local-regional microscopic disease control to the degree that adjuvant radiation may not be needed following $\mathrm{R} 0$ resection. ${ }^{21}$

Whether or not IORT contributes to overall survival was also evaluated in a group of patients treated at the University of Texas M.D. Anderson Cancer Center with neoadjuvant chemoradiation for radiographically resectable pancreatic cancer (no tumor involvement of the celiac axis or superior mesenteric artery with patent portal venous confluence). The authors reported local recurrence to be a component of the first site of failure in $9 \%$ in patients undergoing neoadjuvant fractionated EBRT (30-50 Gy) followed by surgery without IORT compared with $4 \%$ with the addition of IORT. ${ }^{22}$ The contribution of neoadjuvant EBRT to surgery cannot be determined in this study. However, the risk of local recurrence without IORT was too low in this study to see a clinically meaningful benefit in survival. In part due to these results, IORT for resected pancreatic cancer was abandoned at the M.D. Anderson Cancer Center.
It is interesting to contrast these two large experiences using IORT for localized pancreatic cancer. In the current study, positive margins (R1 or R2) occurred in 30 of 83 patients $(36 \%)$ overall, and the local tumor recurrence rate was numerically lower in the higher-risk IORT group $(23 \%$ versus 39\%). Statistical power was limited because of small numbers, but there was a suggestion that IORT improved local tumor control. In contrast, 16 of 132 patients $(12 \%)$ with radiographically resectable tumors that were treated with neoadjuvant chemoradiation had positive margins (all R1) in the M.D. Anderson experience, and the crude local tumor recurrence rate was $4 \%$ with IORT and $9 \%$ without IORT. The absolute differences in the rates of positive margins and local tumor recurrence are most likely the result of differences in patient selection. However, the comparison illustrates the point that statistically nonsignificant improvements in local tumor control have been seen with the addition of IORT to resection and standard adjuvant therapies in cohorts whose combined local recurrence risk was low $(<10 \%$ in the M.D. Anderson study) and moderate $(30-40 \%$ in the current study). However, a local control improvement due to IORT large enough to translate into a survival benefit in pancreatic cancer is probably only possible in a cohort of the highestrisk patients ( $\mathrm{R} 1 / \mathrm{R} 2$ resections) and perhaps even then only in concert with improved systemic therapy. That hypothesis has yet to be tested.

Where does that leave us when considering the treatment of our next patient? IORT appears to reduce or at least delay local tumor recurrence in patients undergoing pancreaticoduodenectomy. The absolute local control benefit depends on the a priori risk of local recurrence. Patients undergoing R0 resection treated with standard adjuvant therapies do not appear to benefit from IORT. IORT should only be considered in patients who are judged to have incompletely resected disease ( $\mathrm{R} 1$ or $\mathrm{R} 2$ resections) intraoperatively. Although a dedicated study of the impact of IORT following R1/R2 resection has not been performed, IORT should continue to be explored in this subgroup in parallel with novel systemic therapies.

\section{REFERENCES}

1. Clarke M, Collins R, Darby S, Davies C, Elphinstone P, Evans E, et al. Effects of radiotherapy and of differences in the extent of surgery for early breast cancer on local recurrence and 15-year survival: an overview of the randomised trials. Lancet. 2005;366:2087-106.

2. Gunderson LL, Willett CG, Harrison LB, Petersen IA, Haddock MG. Intraoperative irradiation: current and future status. Semin Oncol. 1997;24:715-31.

3. Belletti B, Vaidya JS, D'andrea S, Entschladen F, Roncadin M, Lovat $\mathrm{F}$, et al. Targeted intraoperative radiotherapy impairs the stimulation of breast cancer proliferation and invasion caused by surgical wounding. Clin Cancer Res. 2008;14:1325-32. 
4. Tagliabue E, Agresti R, Carcangiu ML, Ghirelli C, Morelli D, Campiglio M, et al. Role of HER2 in wound-induced breast carcinoma proliferation. Lancet. 2003;362:527-33.

5. Formenti SC, Demaria S. Local control by radiotherapy: is that all there is? Breast Cancer Res. 2008;10:215.

6. Demaria S, Ng B, Devitt ML, Babb JS, Kawashima N, Liebes L, et al. Ionizing radiation inhibition of distant untreated tumors (abscopal effect) is immune mediated. Int J Radiat Oncol Biol Phys. 2004;58:862-70.

7. Tepper J, Nardi G, Suit H. Carcinoma of the pancreas: review of MGH experience from 1963 to 1973. Analysis of surgical failure and implications for radiation therapy. Cancer. 1976;37:1519-24.

8. Griffin JF, Smalley SR, Jewell W, Paradelo JC, Reymond RD, Hassanein RE, et al. Patterns of failure after curative resection of pancreatic carcinoma. Cancer. 1990;66:56-61.

9. Hishinuma S, Ogata Y, Tomikawa M, Ozawa I, Hirabayashi K, Igarashi S. Patterns of recurrence after curative resection of pancreatic cancer, based on autopsy findings. J Gastrointest Surg. 2006;10:511-8.

10. Monkemuller K, Fry LC, Malfertheiner P. Pancreatic cancer is 'always non-resectable.' Dig Dis. 2007;25:285-8.

11. Lygidakis NJ, Singh G, Bardaxoglou E, Dedemadi G, Sgourakis G, Nestoridis J, et al. Mono-bloc total spleno-pancreaticoduodenectomy for pancreatic head carcinoma with portal-mesenteric venous invasion. A prospective randomized study. Hepatogastroentergoloy. 2004;51:427-33.

12. Doi R, Imamura M, Hosotani R, Imaizumi T, Hatori $T$, Takasaki $\mathrm{K}$, et al. Surgery versus radiochemotherapy for resectable locally invasive pancreatic cancer: final results of a randomized multiinstitutional trial. Surg Today. 2008;38:1021-8.

13. Neuhaus P, Riess H, Post S, Gellert K, Ridwelski K, Schramm H, et al. CONKO-001: final results of the randomized, prospective, multicenter phase III trial of adjuvant chemotherapy with gemcitabine versus observation in patients with resected pancreatic cancer (PC). J Clin Oncol. 2008;26 (May 20 suppl; abstr LBA4504).
14. Kalser MH, Ellenberg SS. Pancreatic cancer. Adjuvant combined radiation and chemotherapy following curative resection. Arch Surg. 1985;120:899-903.

15. Herman JM, Swart MJ, Hsu CC, Winter J, Pawlik TM, Sugar E, et al. Analysis of fluorouracil-based adjuvant chemotherapy and radiation after pancreaticoduodenectomy for ductal adenocarcinoma of the pancreas: results of a large, prospectively collected database at the Johns Hopkins Hospital. $J$ Clin Oncol. 2008;26:3503-10.

16. Neoptolemos JP, Stocken DD, Friess H, Bassi C, Dunn JA, Hickey $\mathrm{H}$, et al. A randomized trial of chemoradiotherapy and chemotherapy after resection of pancreatic cancer. New Engl J Med. 2004;350:1200-10.

17. Zerbi A, Fossati V, Parolini D. Intraoperative radiation therapy adjuvant to resection in the treatment of pancreatic cancer. Cancer. 1994;73:2930-5.

18. Hiraoka T, Watanabe E, Mochinaga Tashiro S, Miyauchi Y, Nakamura I, et al. Intraoperative irradiation combined with radical resection for cancer of the head of the pancreas. World $J$ Surg. 1984;8:766-71.

19. Sindelar WF, Kinsella TJ. Studies of intraoperative radiotherapy in carcinoma of the pancreas. Ann Oncol. 1999;10(Suppl. 4):S226-30.

20. Reni M, Panucci MG, Ferreri AJ, Balzano G, Passoni P, Cattaneo $\mathrm{GM}$, et al. Effect on local control and survival of electron beam intraoperative irradiation for resectable pancreatic adenocarcinoma. Int J Radiat Oncol Biol Phys. 2001;50:651-8.

21. Marks LB, Prosnitz LR. Postoperative radiotherapy for lung cancer: the breast cancer story all over again? Int J Radiat Oncol Biol Phys. 2000;48:625-7.

22. Breslin TM, Hess KR, Harbison DB, Jean ME, Cleary KR, Dackiw AP, et al. Neoadjuvant chemoradiotherapy for adenocarcinoma of the pancreas: treatment variables and survival duration. Ann Surg Oncol. 2001;8:123-32. 